\title{
Evaluation of the Three-dimensional Welding Residual Stresses Based on the Eigenstran Methodology via X-Ray Measurements
}

\author{
Masaru Ogawa ${ }^{1, a^{*}}$ and Takehiro Ishii ${ }^{2, b}$ \\ ${ }^{1}$ Faculty of Engineering, Yokohama National University, 79-1, Tokiwadai, Hodogaya-ku, \\ Yokohama 240-8501, Japan \\ ${ }^{2}$ Domestic Preventive Maintenance Group, Mitsubishi Hitachi Power Systems, 1-1, Saiwai-cho \\ 3-chome, Hitachi-shi, Ibaraki 317-8585, Japan \\ aogawa-masaru-jv@ynu.ac.jp, btakehiro_ishii@mhps.com
}

Keywords: X-Ray Diffraction, Three-dimensional Residual Stresses, Non-destructive, Inverse Problem, Eigenstrain, Weld

\begin{abstract}
In order to predict the lifetime of welded structures, it is important to evaluate threedimensional residual stresses nondestructively. However, X-ray diffraction is useful for measuring residual stresses only on the surface. Neutron diffraction is known to be an effective nondestructive method for measuring residual stresses till a depth of several tens of millimeters. Nevertheless, it is not a technique useful for on-site measurements. This is because neutron diffraction is achievable only within special irradiation facilities. In this paper, the authors have proposed a non-destructive method to estimate three-dimensional residual stresses by using X-ray measurements. In this method, the residual stresses for an entire structure are calculated from eigenstrains by using an elastic FEM (Finite Element Method) analysis. Eigenstrains can be estimated from elastic strains measured through X-ray diffraction using inverse analysis. The relationship between the three-dimensional eigenstrains and the surface elastic strains can be obtained if the Young's modulus, Poisson's ratio, and dimensions of the structure are known. This study aims to demonstrate the effectiveness of the proposed method on an actual butt-welded plate made of stainless steel SUS430. To evaluate the accuracy of this method, the residual stresses estimated using the method was compared with that measured using X-ray diffraction. In order to maximize the estimation accuracy of this inverse analysis, we considered the eigenstrain distributions along both the welding and thickness directions, as well elastic strains measured on the weld metal were used to estimate.
\end{abstract}

\section{Introduction}

It is important to predict crack propagation for observed cracks to prevent fatigue fractures and stress corrosion cracking (SCC) in welded structures. However, it is difficult to estimate crack growth rate without information on welding residual stresses. Three-dimensional welding residual stresses can be simulated qualitatively by using thermal elastic-plastic FEM analysis [1,2]. Nevertheless, it is recommended that each welded structure be quantitatively measured because individual differences in welding residual stress between them can be high. Currently, diffraction methods, including X-ray diffraction and neutron diffraction $[3,4]$, are used as nondestructive measurement methods. However, it is impossible to measure three-dimensional stress distribution on-site by using these methods. Xray diffraction is useful to measure only surface residual stresses. Higher energy diffraction methods, including synchrotron and neutron diffractions, are available only within special irradiation facilities. Therefore, the targeted welded parts have to be destructively cut from an actual welded structure for measuring stress distribution. The cutting process releases some of the residual stresses, which affects the accuracy of the entire measurement process.

As an alternative, the Bead Flush method, which is based on the eigenstrain methodology [5], has been proposed [6]. In this method, three-dimensional residual stresses are calculated by conducting 
elastic FEM analysis on eigenstrains, which in turn are estimated through inverse analysis [7] on released strains measured using strain gauges during the removal of the weld reinforcement. Eigenstrains are defined as a sum of inelastic strains [5], but they are different from the sum of physical inelastic strains such as plastic, thermal, and transformation strains [8]. Here, the smoothening of excess weld metal can be regarded as a non-destructive process essentially because it can be eliminate a toe of weld that has the potential to become a crack starter. However, processing strains that arise on the machined surface owing to this method can worsen the accuracy of strain gauges that measure released strains.

In order to overcome the disadvantages of the Bead Flush method, the first author has proposed the use of X-ray diffraction instead of strain gauges to estimate both the welding eigenstrains and processing strains [9]. The effectiveness of this method has been proved via numerical simulation. Besides, experimental demonstrations of this method have been conducted on an actual butt-welded plate made of $2 \mathrm{Cr}-1 \mathrm{Mo}$ ferritic steel [10]. However, the accuracy of estimating residual stresses along the perpendicular to the welding direction is not sufficient. Numerical simulation has confirmed the higher accuracy of this method when residual strains on machined surface measured by X-ray diffraction are used for estimation [11].

In the present study, the authors have estimated residual stresses of welded plates made of SUS430 by using this method on elastic strains measured over the weld metal [12]. For this estimation, it is assumed that welding eigenstrains are constant along the thickness direction even though the butt-welded joint has single U-groove before welding. The eigenstrain distribution along the welding direction is also neglected during inverse analysis. The residual stresses of welded joints made of SUS430 are estimated using this method after considering eigenstrain distributions along both the welding and thickness directions. Parameters to express eigenstrain distributions are determined following the Response Surface methodology. To evaluate the estimation accuracy, the residual stresses estimated using this method are compared with those measured by X-ray diffraction.

\section{Analytical Procedure}

The elastic strains $\left\{\varepsilon_{\mathrm{e}}\right\}$ of the concerned elements and the welding eigenstrains $\left\{\varepsilon^{*}\right\}$ for the entire structure can be related through an intermediary - an elastic response matrix $\left[\boldsymbol{R}_{\mathrm{e}}\right]$ as:

$$
\left\{\boldsymbol{\varepsilon}_{\mathrm{e}}\right\}=\left[\boldsymbol{R}_{\mathrm{e}}\right]\left\{\boldsymbol{\varepsilon}^{*}\right\}
$$

The $i$-th column of the response matrix can be determined by imposing a unit eigenstrain on the $i$-th component of $\left\{\varepsilon_{\mathrm{e}}{ }^{*}\right\}$ during elastic FEM analysis. Based on Eq. (1), the surface elastic strains before and after the removal of the weld reinforcement - $\left\{\varepsilon_{\mathrm{eb}}\right\}$ and $\left\{\varepsilon_{\mathrm{ea}}\right\}$ respectively - can be expressed, as follows:

$$
\begin{aligned}
& \left\{\boldsymbol{\varepsilon}_{\mathrm{eb}}\right\}=\left[\boldsymbol{R}_{\mathrm{b}}\right]\left\{\boldsymbol{\varepsilon}^{*}\right\} \\
& \left\{\boldsymbol{\varepsilon}_{\mathrm{ea}}\right\}=\left[\boldsymbol{R}_{\mathrm{a}}\right]\left\{\boldsymbol{\varepsilon}^{*}\right\}+\left[\boldsymbol{R}_{\mathrm{a}}\right]\left\{\boldsymbol{\varepsilon}_{\mathrm{pa}}{ }^{*}\right\}
\end{aligned}
$$

where $\left[\boldsymbol{R}_{\mathrm{b}}\right]$ and $\left[\boldsymbol{R}_{\mathrm{a}}\right]$ respectively denote the response matrix before and after the removal. $\left\{\boldsymbol{\varepsilon}_{\mathrm{pa}}{ }^{*}\right\}$ is the processing strain that remains after the smoothening. By jointing Eq. (2) and Eq. (3), we obtain the following equations [18]:

$$
\begin{aligned}
& \left\{\boldsymbol{\varepsilon}_{\mathrm{eb}} \boldsymbol{\varepsilon}_{\mathrm{ea}}\right\}^{\mathrm{T}}=[\boldsymbol{R}]\left\{\boldsymbol{\varepsilon}^{*} \boldsymbol{\varepsilon}_{\mathrm{pa}}\right\}^{*} \\
& {[\boldsymbol{R}]=\left[\begin{array}{cc}
\boldsymbol{R}_{\mathrm{b}} & \mathbf{0} \\
\boldsymbol{R}_{\mathrm{a}} & \boldsymbol{R}_{\mathrm{a}}
\end{array}\right]}
\end{aligned}
$$

Actual nondestructive measurements of elastic strains contain observational errors $\left\{\boldsymbol{\varepsilon}_{\mathrm{err}}\right\}$, as given below: 


$$
\left\{\boldsymbol{\varepsilon}_{\mathrm{ebm}} \boldsymbol{\varepsilon}_{\mathrm{eam}}\right\}^{\mathrm{T}}=[\boldsymbol{R}]\left\{\boldsymbol{\varepsilon}^{*} \boldsymbol{\varepsilon}_{\mathrm{pa}}\right\}^{\mathrm{T}}+\left\{\boldsymbol{\varepsilon}_{\mathrm{err}}\right\}
$$

where $\left\{\varepsilon_{\mathrm{ebm}}\right\}$ and $\left\{\varepsilon_{\mathrm{eam}}\right\}$ are both elastic strains measured using nondestructive methods. The estimated values of welding eigenstrains $\left\{\boldsymbol{\varepsilon}_{\text {est }}{ }^{*}\right\}$ and processing strains $\left\{\boldsymbol{\varepsilon}_{\text {pa }}{ }_{\text {est }}{ }^{*}\right\}$ can be obtained from elastic strains measured by X-ray diffraction, through inverse analysis, as shown below:

$$
\left\{\boldsymbol{\varepsilon}_{\text {est }}{ }^{*} \boldsymbol{\varepsilon}_{\text {pa_est }}\right\}^{*}=[\boldsymbol{R}]^{+}\left\{\boldsymbol{\varepsilon}_{\text {ebm }} \boldsymbol{\varepsilon}_{\text {eam }}\right\}^{\mathrm{T}} .
$$

where $[\boldsymbol{R}]^{+}$is the Moore and Penrose generalized inverse matrix [7] of $[\boldsymbol{R}]$, which is given by:

$$
[\boldsymbol{R}]^{+}=[\boldsymbol{R}]^{\mathrm{T}}[\boldsymbol{R}]\left([\boldsymbol{R}]^{\mathrm{T}}[\boldsymbol{R}][\boldsymbol{R}]^{\mathrm{T}}[\boldsymbol{R}]\right)^{-}[\boldsymbol{R}]^{\mathrm{T}} .
$$

\section{Target Joint and Measurements by X-ray Diffraction}

The dimensions of the target plate (Fig. 1) were $80 \times 100 \times 10 \mathrm{~mm}^{3}$. The widths of the weld bead on the top and bottom surfaces were $16 \mathrm{~mm}$ and $8 \mathrm{~mm}$, respectively. The welding conditions are listed in Table 1. Ferritic weld wires were used to join stainless steel SUS430 to enable easier measurements over the weld metal by using X-ray diffraction. It took nine weld passes to fill single U-groove. Except during the first weld pass, the heat source was oscillated to avoid poor penetration along the boundaries between the base and weld metals. The excess metal on the top surface was removed using a flat-surface grinding machine.

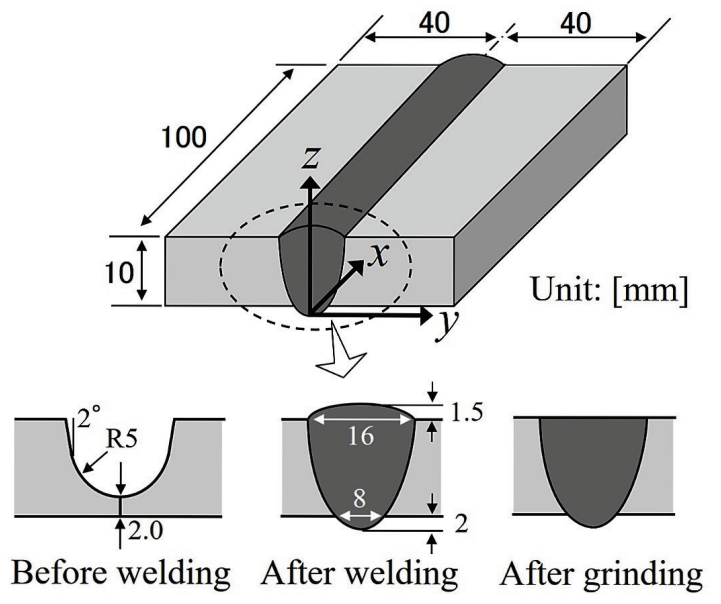

Fig. 1 Butt-welded
Table 1 Welding conditions.

\begin{tabular}{|l|l|}
\hline Welding method & TIG \\
\hline Welding current & $200 \mathrm{~A}$ \\
\hline Welding voltage & $10 \mathrm{~V}$ \\
\hline Welding speed & $10 \mathrm{~cm} / \mathrm{min}$ \\
\hline Welding electrodes & $\mathrm{YT}-160$ \\
\hline Diameter of electrodes & $2.4 \mathrm{~mm}$ \\
\hline Shield gas & $\mathrm{Ar}$ \\
\hline Preheating & $\mathrm{No}$ \\
\hline Inter-pass temperature & Under $150^{\circ} \mathrm{C}$ \\
\hline Welding oscillation & Yes \\
\hline
\end{tabular}

Elastic strains along the welding direction and the perpendicular to the welding direction on the top surface were measured using X-ray diffraction to estimate welding eigenstrains. Fig. 2 shows the measurement points before and after the removal of weld reinforcement. Measurement conditions of $\mathrm{X}$-ray diffraction were typical, as shown in Table 2. This X-ray diffraction is based on the $\sin ^{2} \psi$ method. Irradiation area of X-ray diffraction was set at $2 \times 2 \mathrm{~mm}^{2}$, as normal range. Note that $x, y$, and $z$ are the welding direction, the perpendicular to the welding direction from the center of the weld, and the thickness direction, respectively, as shown in Fig. 1.

\section{Stabilizing Solution in Inverse Analysis}

FEM Model. An FEM model used in this estimation is shown in Fig. 3. This model is symmetric about the weld line. The numbers of nodes and elements before the removal of the excess metal are 1397 and 1020, respectively. The Young's modulus and Poisson's ratio were set at $200 \mathrm{GPa}$ and 0.3 , respectively. For FEM analysis, the commercial software ANSYS (Cybernet Systems Company, Limited, Japan), was used.

Stabilizing Solutions. Solutions obtained from this inverse analysis are relatively sensitive because the three-dimensional eigenstrain distributions are being estimated from measurements of 
two-dimensional surface strains that include observational errors. During this study, the method of functional approximation of welding eigenstrains and the artificial noise method [7] were introduced. These are described below.

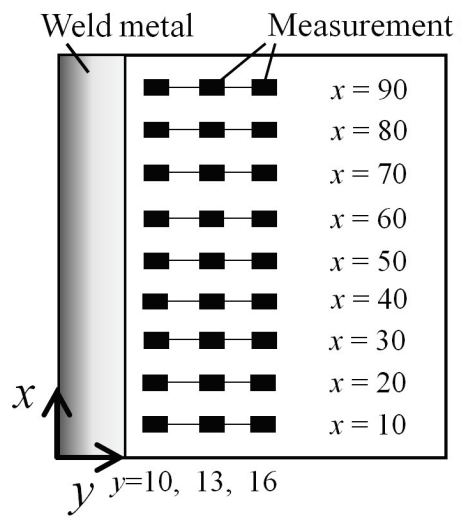

(a) Before the removal.

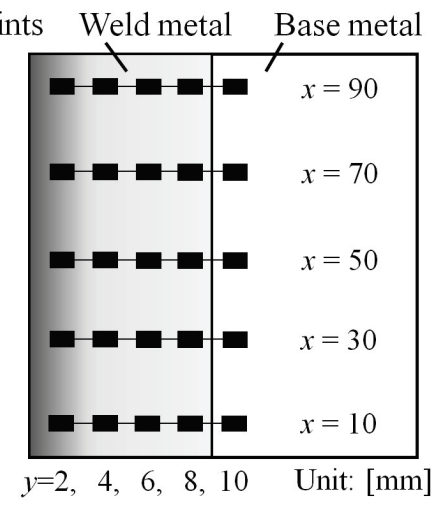

(b) After the removal.

Fig. 2 Measurement points before and after the removal.

Table 2 Measurement conditions.

\begin{tabular}{|l|l|}
\hline Target & $\mathrm{Cr}$ \\
\hline Tube voltage and current & $40 \mathrm{kV}, 200 \mathrm{~mA}$ \\
\hline Bragg's angle $\left(2 \theta_{0}\right)$ & $156^{\circ}$ \\
\hline Incident angles $(\Psi)$ & $0,18.4,26.7,33.2,39.2,45.0^{\circ}$ \\
\hline
\end{tabular}
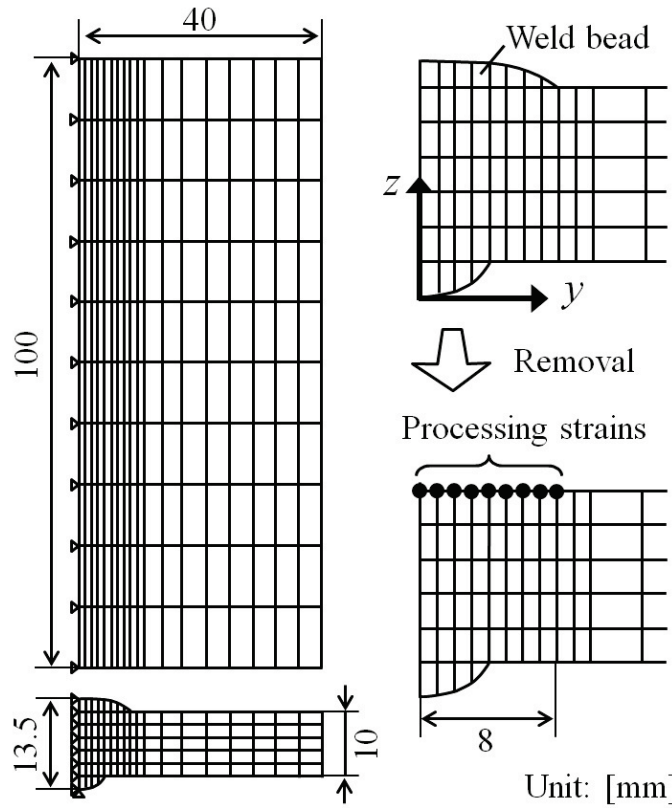

Processing strains

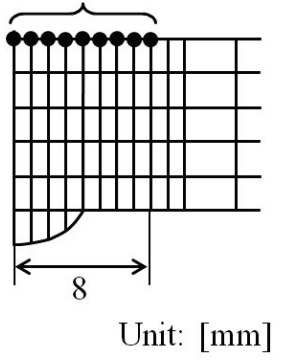

Fig. 3 An FEM model before the removal.

First, logistic functions were used to express welding eigenstrains [13], as shown below:

$$
\left\{\boldsymbol{\varepsilon}_{s}^{*}\right\}(y)=\sum_{i=1}^{4} \frac{\left\{\boldsymbol{a}_{s i}\right\}}{1+\exp \left(p+q_{i} y\right)}, \quad p=-5.0, q_{1}=0.60, q_{2}=0.40, q_{3}=0.30, q_{4}=0.25
$$

where the subscript $s$ denotes any of the three directions - welding $(x)$, perpendicular to the welding $(y)$ and thickness $(z)$ directions. The constants $p$ and $q_{i}$ are determined such that eigenstrains are distributed within $40 \mathrm{~mm}$ in the $y$ direction [14]. Therefore, the number of elements in the unknown vector $\left\{\boldsymbol{a}_{\mathrm{si}}\right\}$ becomes 12 (four functions in each direction). In the previous study, it was assumed that eigenstrains were not distributed along the welding and thickness directions [12]. In order to improve the estimation accuracy in this study, eigenstrain distributions in these directions were considered using the equation below instead of Eq. (9) [10].

$$
\left\{\boldsymbol{\varepsilon}_{s}^{*}\right\}(x, y, z)=\sum_{i=1}^{4} \frac{\left\{\boldsymbol{a}_{s i}\right\}(\beta+1)}{1+\exp \left(p+q_{i} \sqrt{y^{2}+(t-z)^{2} / \alpha}\right)}
$$

where it is better to set constant $t$ as the gross thickness that includes bead heights on the top and bottom. In this study, the value of $t$ was set at $14 \mathrm{~mm}$. The most probable values of $\alpha$ and $\beta$ are determined based on the Response Surface methodology. Additionally, it was assumed that processing strains formed on the machined surface were constant along the welding direction, and that their values from $y=0 \mathrm{~mm}$ to $2 \mathrm{~mm}$ and from $3 \mathrm{~mm}$ to $8 \mathrm{~mm}$ are equal. Consequently, the total number of unknown parameters of the processing strains was 6 ( 2 surfaces in each direction).

The other approach to improve well-posedness of this inverse analysis is the used of the artificial noise method [7]. When the response matrix $[\boldsymbol{R}]$ with rank $n$ is decomposed, the following equations can be obtained. 


$$
\begin{aligned}
& {[\boldsymbol{R}]^{+}=[\boldsymbol{U}][\boldsymbol{B}]^{-}[\boldsymbol{V}]^{\mathrm{T}}} \\
& {[\boldsymbol{B}]^{-}=\left[\begin{array}{ll}
\boldsymbol{B}_{n}^{-} & \boldsymbol{0} \\
\boldsymbol{0} & \boldsymbol{0}
\end{array}\right], \quad\left[\boldsymbol{B}_{n}\right]^{-}=\left[\begin{array}{cccc}
1 / \mu_{1} & & & 0 \\
& 1 / \mu_{2} & & \\
& & \ddots & \\
0 & & & 1 / \mu_{n}
\end{array}\right] \mu_{1} \geqq \mu_{2} \geqq \ldots \geqq \mu_{n} \geqq 0 .}
\end{aligned}
$$

If the absolute value of the singular value $\mu_{j}(1 \leqq j \leqq n)$ reduces, measurement errors exert a larger effect on solutions. To eliminate this influence in the artificial noise method, $\left[\boldsymbol{B}_{n_{-}}\right]^{-}$is used instead of $\left[\boldsymbol{B}_{n}\right]^{-}$, as shown below:

$$
\left[\boldsymbol{B}_{n \_\gamma}\right]^{-}=\left([\boldsymbol{B}]^{2}+\gamma[\boldsymbol{I}]\right)^{-}[\boldsymbol{B}]^{\mathrm{T}}
$$

where $[\boldsymbol{I}]$ denotes a unit matrix. Solutions can be stabilized by increasing the value of the real number $\gamma$. In this inverse analysis, the L-curve method [15] was applied to determine the most probable value of $\gamma$.

\section{Estimated Results}

The most probable values of $\alpha$ and $\beta$ were

Residual norm determined from a response surface (Fig. 4) to be 0.7 and 0.010 , respectively. The vertical axis in this graph is a residual norm that is a difference between the estimated and measured residual strains on the measurement points. Therefore, the most probable values can be found at the minimum value.

The residual stresses at $x=50 \mathrm{~mm}$ on the bottom surface estimated using this method are compared with residual stresses directly measured using X-ray diffraction (Fig. 5). The dashed green lines are the estimated values without considering eigenstrain distributions in the $x$ and $z$ directions. The residual stresses, estimated using $\alpha$ and $\beta$, are drawn by

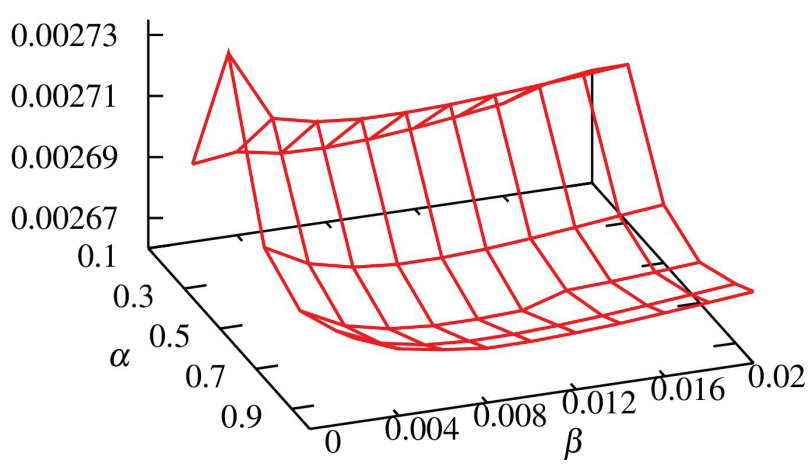

Fig. 4 A response surface to determine the most probable values of $\alpha$ and $\beta$. using solid blue lines. An almost equivalent estimation accuracy can be observed at $x=50 \mathrm{~mm}$ on the bottom surface (Fig. 5(a)). On the other hand, estimation accuracy can be improved at $y=10 \mathrm{~mm}$ by considering eigenstrain distributions (Fig. 5(b)).

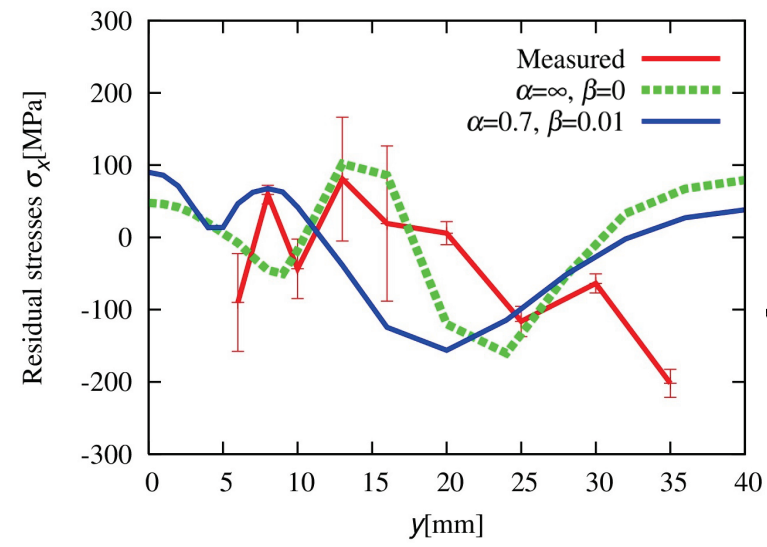

(a) At $x=50 \mathrm{~mm}$.

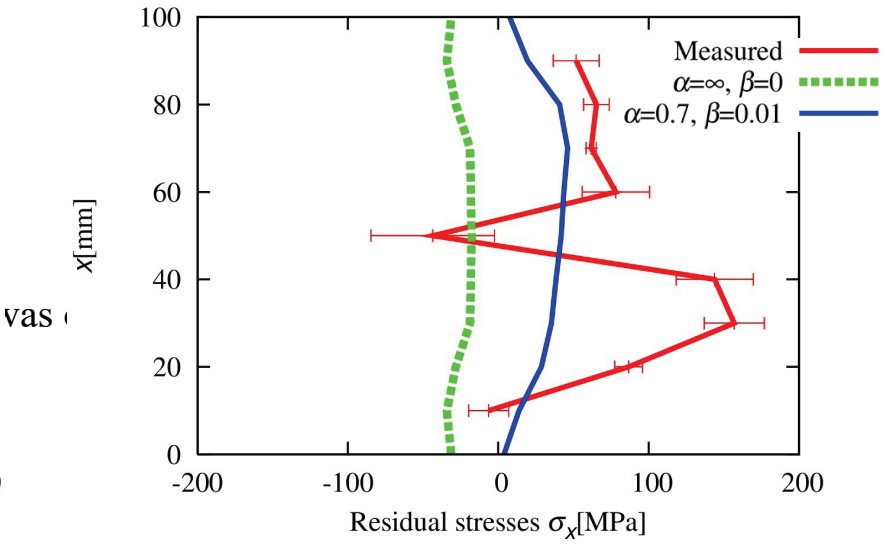

(b) At $y=10 \mathrm{~mm}$.

Fig. 5 Exact and estimated residual stresses along the $x$ direction on the bottom surface. The red lines are measured values using X-ray diffraction. Estimated residual stresses with and without considering eigenstrain distributions are drawn by the solid blue line and the broken green line, resnectivelv, 
However, it might be better to use higher-degree equation for the approximation because residual stresses measured by X-ray diffraction were largely oscillated along the welding direction, as Fig. 5(b). Eigenstrain distributions might be complicated by the oscillation of the heat source during welding.

\section{Summary}

The method that is based on the eigenstrain methodology and X-ray diffraction was applied on an actual butt-welded joint made of SUS430 to evaluate three-dimensional residual stresses. The estimation accuracy of this method was improved by considering the eigenstrain distributions along the welding and thickness directions.

\section{Acknowledgements}

The authors acknowledge Tonen General Sekiyu Research/Development Encouragement \& Scholarship Foundation and Mazak Foundation for their generous financial assistance. As well, this work was supported by JSPS KAKENHI Grant Number 26820005. We would also like to thank Editage for English language editing.

\section{References}

[1] Z. Saternus, W. Piekarska, M. Kubiak, T. Domanski and L. Sowa, Procedia Engineering 136 (2016) 95-100. http://dx.doi.org/10.1016/j.proeng.2016.01.180

[2] Y. Ye, J. Cai, X. Jiang, D. Dai and D. Deng, Advances in Engineering Software 86 (2015) 3948. http://dx.doi.org/10.1016/j.advengsoft.2015.04.001

[3] A. Skouras, A. Paradowska, M.J. Peel and M.J. Pavier, International Journal of Pressure Vessels and Piping 101 (2013) 143-153. http://dx.doi.org/10.1016/j.ijpvp.2012.11.002

[4] H.J. Kirkwood, S.Y. Zhang, A.S. Tremsin, A.M. Korsunsky, N. Baimpas and B. Abbey, Materials Today: Proceedings 2S (2015) S414-S423.

[5] T. Mura, Micromechanics of Defects in Solids, Martinus Nijhoff Publishers, 1987. http://dx.doi.org/10.1007/978-94-009-3489-4

[6] H. Nakamura, Y. Naka W. Park and H. Kobayashi, Current Topics in Computational Mechanics, ASME PVP, 305 (1995) 49-56.

[7] S. Kubo, Inverse Problems, Baifukan, 1992 (in Japanese).

[8] K. Masuda and H. Nakamura, The Japan Society of Mechanical Engineers, Series-A, 76 (2010) 884-892 (in Japanese).

[9] M. Ogawa, The Japan Society of Mechanical Engineers, Series-A, 79 (2013) 1266-1277 (in Japanese).

[10] M. Ogawa, T. Ishii and S. Furusako, Journal of the Society of Materials Science, Japan, 64 (2015) 932-939 (in Japanese). http://dx.doi.org/10.2472/jsms.64.932

[11] M. Ogawa, International Journal of Computational Materials Science and Engineering, 3 (2014) 1450023. http://dx.doi.org/10.1142/S2047684114500237

[12] M. Ogawa and T. Ishii, Proceedings of the International Conference on Advanced Technology in Experimental Mechanics 2015, (2015) 36.

[13] K. Kumagai, H. Nakamura and H. Kobayashi, The Japan Society of Mechanical Engineers, Series-A, 65 (1999) 133-140 (in Japanese).

[14] Y. Ueda and N. X. Ma, Japan Welding Society, 11 (1993) 189-195 (in Japanese).

[15] P. C. Hansen, SIAM Review, 34 (1992) 561-580. 